\title{
MSBE Analysis with Frequency Spectra for Automated Identification of Epileptic Seizure
}

\author{
Hemlata Pal
}

\begin{abstract}
Objective-This study introduces a reliable automated seizure detection technique based on MSBE (Multi scale bubble entropy) and frequency spectral analysis. Method- This paper aims to develop a novel seizure detection technique that incorporates AM FM model for decomposition of EEG into different sub bands. In our approach, integrated feature set is constructed using multi scale bubble entropy analysis at each sub band and frequency spectral analysis at each electrode. Result-In this paper, an application of bubble entropy with different frequency parameter such as PPF and PSD is provided in order to access its stable and outstanding performance on epileptic seizer detection. The experimental results show that classification accuracy is improved with this algorithm. These finding suggest that extracted features can be used for treatment of epilepsy. Significance- This method provides greater stability and discriminative power, so this technique could be used to detect wider range of seizures.
\end{abstract}

Keywords: Epilepsy, EEG, Multi scale bubble entropy, Power spectral density, Seizure detection

\section{INTRODUCTION}

Epilepsy is a serious neurological disorder and it is associated with spontaneous debilitating seizers. EEG recording is required for definitive diagnosis of epilepsy. EEG is basically the multivariate signal which is acquired by the use of sensors and mounting electrodes to provide electrical metrics of brain. EEG is the bioelectric signal and it represents the summation of electrical activities of millions of neurons. This bioelectric signal has great potential in helping us to understand the underlying state of human brain.

Existing approaches for detection of epileptic seizures can broadly classified on the basis of linear features extraction and nonlinear feature extraction. There have been several methods for prediction of seizures using linear features based on spectral analysis such as PSD, Peak frequency, Spectral edge frequency, relative power and energy [1], [5], [9], [23], [24], [25] which provides basic information, but they usually exhibit common drawback of inconsistent performance. In another category of approaches that exploits nonlinear characteristics of EEG, such as HOS, correlation and entropy [2], [6], [7], [16].

Entropy has been extensively used for computation of features from EEG for auto- mated diagnosis of epilepsy [3] [8], [13]. Several research groups have also worked on method which involves features computation of entropy at multiple scales [17], [18]. This entropy analysis performed feature extraction using sample entropy at multiple scales and is able to provide more detailed information of EEG signal. Some of these entropy measures can have some limitations such as scale factor ( $r$ ) and embedding dimension (n) to discriminate EEG signal of different complexity. Another recently developed approach termed as bubble entropy analysis [19], computes bubble entropy based on permutation entropy. This method is almost free of the parameter that means it removes the requirement of scale factor and minimizes the importance of embedding dimension for analysis of EEG signal. It provides remarkable stability at original time scale.

In this paper, we make effort to explore a novel application of MSBE (Multi scale bubble entropy) analysis with different frequency parameters (PPF \& PSD) suitable for epilepsy detection by employing integrated feature set.

1) At first, AM FM model is engaged that can decompose an EEG signal according to its frequency content and MSBE analysis is performed to achieve remarkable stability and discriminating capability.

2) Second, frequency spectra analysis is performed to identify PPF and PSD quantities from EEG signal.

3) Application of an integrated feature vectors to produce intelligent classification working program.

In the rest of paper, Section II outlines the overview of related literature. Section III presents the proposed methodology used for seizure detection. Experimental results and discussion, including comparison with existing method, are addressed in section IV. The concluding remarks are given in section $\mathrm{V}$.

\section{AN OVERVIEW OF RELATED LITERATURE}

Time domain analysis-Subasi (4), used DWT for decomposition of EEG signals. Four features are extracted from wavelet coefficients. These features are average power, mean of the absolute value, ratio of absolute mean value and standard deviation. R. Yadav [22] present morphology based seizure detection. It uses the sharpness of waveform as feature for detection of seizure. 


\section{MSBE Analysis with Frequency Spectra for Automated Identification of Epileptic Seizure}

Time \& Frequency analysis -Ali Shahidi Zandi et al., [23] proposed a novel wavelet-based algorithm for identification of seizures. In this method, combined seizure index (CSI) is used as feature for detection of seizure and CSI is based on both rhythmicity and relative energy of each epoch. Khan and Gotman [1] presented a seizure detection technique to characterize epileptic seizures using wavelet analysis. Three different features are extracted and these are relative average amplitude, coefficient of variation of amplitude, and relative scale energy. Mitra et al. [25] proposed a multistage seizure detection scheme. In this technique various morphological components are used as feature for detection of seizures.V. Srinivasan at el. [5] deals with automatic seizure detection method using an Elman network (EN). This method makes use of five different attributes to detect the epileptic seizures, it considers two time-domain (spike rhythmicity and relative spike amplitude) and three frequency-domain features (dominant frequency, average power in the main energy zone and normalized spectral entropy) of the EEG signal. Alexandros at el. [9], performed $t$ - $f$ analysis for determination of epileptic seizures from EEG segment. The $t-f$ analysis of EEG is performed and then power spectrum density (PSD) is determined.

HOS analysis- N. Kannathal et al. [2] and Ghose Dasidas et al. [6], [7] developed accurate and reliable seizure detection algorithm using "Non linear features" analysis. Chua at el. [11], [12] Proposed a novel algorithm based on HOS features. The extracted features are applied to GMM model for classification of EEG. Acharya at el. [16], deals with two measures, called entropy and HOS for characterization of epileptic seizures.

Entropy analysis - V. Srinivasan at el. [8] discussed two different types of classifier for the determination of epileptic seizures. These are Elman network and probabilistic neural networks. It uses approximate entropy (ApEn) as feature for detection of seizures. N. Kannathal [3] basically deals with different entropy parameter for classification of EEG and it also provides comparison of the different entropy estimator. In this analysis, two entropy estimators are considered and they are spectral entropies and embedding entropies. ANFIS (Adaptive Neurofuzzy Inference System) classifier is used for classification purpose. Hasan ocak at el.[13] performed the detection of epileptic seizure on the basis of discrete wavelet transform and approximate entropy. Bhattavharyya et al. [17], [18] use Multi scale entropy analysis for detection of seizures.

\section{Proposed METhodology}

\section{A. AM FM model}

In our methodology, AM-FM model is used for decomposition of EEG signal into different frequency spectral bands. These frequency spectral bands are delta, alpha, theta, beta and gamma which are commonly used for detection of seizure. The frequency band that contains information relative to seizure detection task lies in the frequency spectral range of $0-40 \mathrm{~Hz}$. EEG signals are multi component signal that can be considered as superposition of mono component signal. Each EEG epoch can be represented by AM-FM model and being characterized by amplitude parameter and phase parameter. Therefore multichannel EEG signal could be formulated as summation of mono component EEG epochs of $E_{y}(n)$ and it can be defined as follows:.

$$
E(n)=\sum_{y=1}^{n} A_{y}(n) \cos \Psi_{y}(n)+N_{y}
$$

Where $A_{y}(n)$ is the instantaneous amplitude component, $\Psi_{\mathrm{y}}(\mathrm{n})$ represent the instantaneous phase component and $\mathrm{N}_{\mathrm{y}}$ is the additive noise and error.

\section{B. Feature extraction}

It is well documented that frequency spectral analysis of EEG signal of different band is correlated with affective state of human brain. Therefore we consider the frequency spectral analysis first for feature computation and then entropy analysis is performed for complexity measurement. First, the algorithm extracts features from frequency spectral analysis of EEG such as PSD (Power spectral analysis density), and PPF (Peak Power Frequency). Then we perform multi scale bubble entropy analysis under different sub band.

PPF - Peak Power Frequency is defined as the frequency at which the peak power occurs. PPF have good discriminating capability, so that we consider it as one of the feature for detection of seizure. PPF is given as:

$$
\mathbf{F}_{\mathrm{PP}}=\arg \left\{\frac{\mathrm{fsam}}{N} \max _{j=0}^{N-1} P(j)\right.
$$

In the above equation $\mathbf{f}_{\text {sam }}$ and $\mathbf{N}$ represent the sampling frequency and number of samples respectively. Peak power frequency is determined from nineteen region of brain for computation of first feature set $\left(\mathrm{F}_{\mathrm{PPF}}\right)$. $\mathrm{F}_{\mathrm{PPF}}$ can be represented as:

$$
\left[F_{P P F}=F_{P \delta 1}, F_{P \theta 1}, F_{P \alpha 1}, F_{P \beta 1} \ldots F_{P \delta 19}, F_{P \theta 19}, F_{P \alpha 19}, F_{P \beta 19}\right]
$$

PSD- Power spectral density basically represents the distribution of signal's power over frequency of complex EEG signal. It provides frequency content of EEG. Power spectral density is given as fast fourier transform (FFT) of autocorrelation function of sequence of complex EEG signal.

\section{Bubble entropy}

Bubble entropy is defined as entropy which is almost free of parameters. Bubble entropy provides higher stability because of two key features: 1) Elimination of necessity of scale factor (r). 2) Minimize the importance of embedding dimension ( $\mathrm{m}$ ). Bubble entropy is basically based on combination of conditional permutation entropy and renyi's entropy. Initially, it is computed from permutation entropy, but they usually exhibit common drawback of unconditional and non descriptive performance. Permutation entropy is given as:

$$
\mathbf{E}_{\mathrm{pe}}=-\sum_{k=1}^{i} p(J k) \log (\mathrm{p}(\mathrm{Jk}))
$$

$\mathrm{J}_{\mathrm{k}}$ is time series of vectors which is constructed using sorting process from EEG.

To make the output performance to be more descriptive, renyi's entropy is also used. Renyi's entropy is defined as:

$$
\mathbf{E}_{\mathbf{R}}=-\log \sum_{\tilde{k}=1}^{\tilde{i}} p(k)^{2}
$$


Therefore a combination of permutation and renyi's entropy is used which provides both conditional and descriptive metric. Conditional renyi's permutation entropy is given as:

$$
E_{\text {CRpeN }}=\left(E_{R}^{n+1}-E_{R}^{n}\right) / \log (n+1)
$$

Where the factor $[1 / \log (n+1)]$ is used for normalization purpose and $n$ is the embedding dimension.

Bubble entropy analysis utilize bubble sort algorithm and count the number of swaps necessary to sort vectors in ascending order and then computes CRPeN entropy from EEG signal at multiple scales.

$$
E_{B}=\left(E_{\text {swaps }}^{n+1}-E_{\text {swaps }}{ }^{n}\right) / \log (\mathbf{n}+1 / n-1)
$$

Multi scale bubble entropy analysis is performed for construction of third feature set. $\left(\mathrm{F}_{\mathrm{MSBE}}\right)$ can be given as:

$$
\left[F_{\mathrm{MSBE}}=\mathrm{F}_{\mathrm{BE} \delta}, \mathrm{F}_{\mathrm{BE \theta}}, \mathrm{F}_{\mathrm{BE} \alpha}, \mathrm{F}_{\mathrm{BE} \beta}, \mathrm{F}_{\mathrm{BE} \gamma}\right]
$$

Finally, all three parameter set are used to construct final feature vector $\left(\mathrm{F}_{\mathrm{T}}\right) . \mathrm{F}_{\mathrm{T}}$ is defined as:

$$
\left[F_{\mathrm{T}}=\mathbf{F}_{\mathrm{PPF}}+\mathbf{F}_{\mathrm{PSD}}+\mathbf{F}_{\mathrm{MSBE}}\right]
$$

\section{EXPERIMENTAL RESULTS AND DISCUSSION}

\section{A. Database}

In this paper, we have used EEG database provided by university of Bonn, Germany. This database contains normal EEG signal from healthy subjects as well as ictal and pre ictal EEG signal acquired from epileptic patient. The sampling frequency of EEG signal is $173.61 \mathrm{~Hz}$ and having $12 \mathrm{bit} \mathrm{A} / \mathrm{D}$ resolution. The signals were recorded by 128 channel amplifier system and provides frequency band of $0-40 \mathrm{~Hz}$ which contain all seizure event.

\section{B. Results}

In this approach, we investigated the use of MSBE with the frequency spectral parameter (PPF and PSD) for detection of epileptic seizure activity from EEG signal. 3D view of brain is shown in fig 1 . The fig 2 shows the obtained raw EEG dataset of seizure, pre seizure (Abnormal) and normal period. Although this figure show that patient experienced major changes during pre seizure period. Amplitude based DSA of EEG data is also shown in figure 3. Monitoring results of PPF at each electrode are shown in fig 4 and fig 5. In these figure, blue, green and red bars are used to represent normal, pre seizure and seizure event respectively. Compared to seizure and normal event, majority of the PPF parameter attains higher values for pre seizure event.

The PSD graphs obtained for different classes of EEG are presented in fig. 6 , fig. 7 and fig 8 . The fig. 6 shows that power spectral density of normal segment looks more stable and attains lower peaks. Furthermore, higher peaks can be seen in the seizure and pre seizure period. These PSD graphs are unique for each class and hence can be used for distinguishing different classes of EEG.

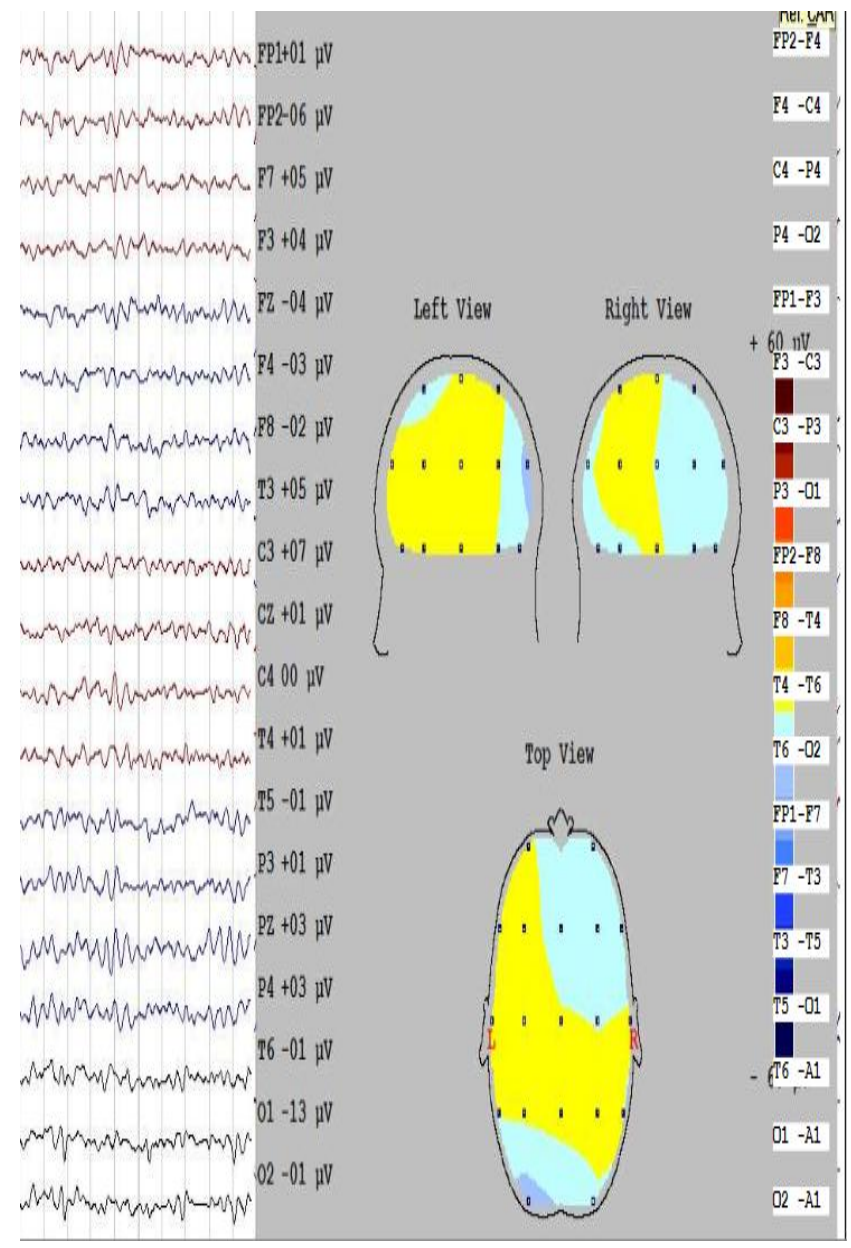

Fig.1. 3D view of brain

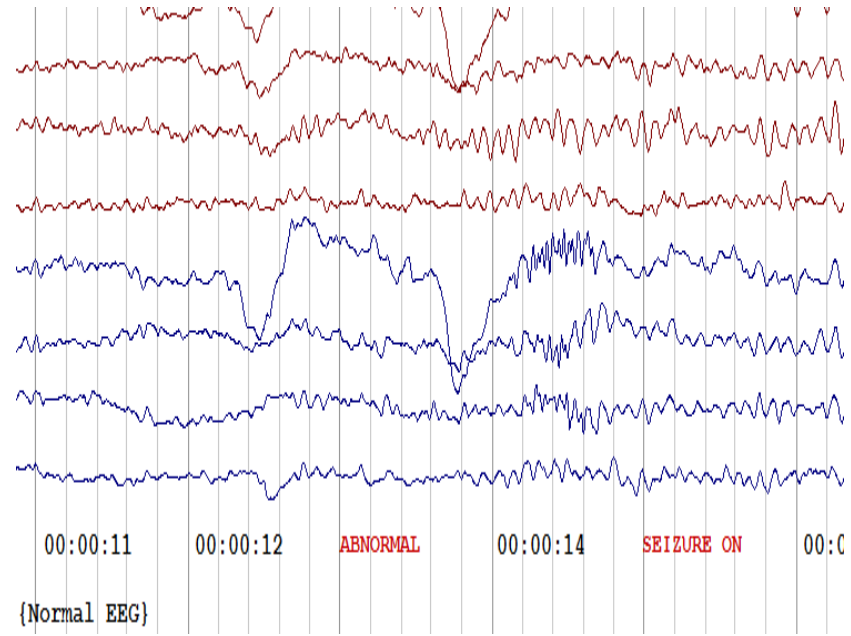

Fig.2. EEG segment

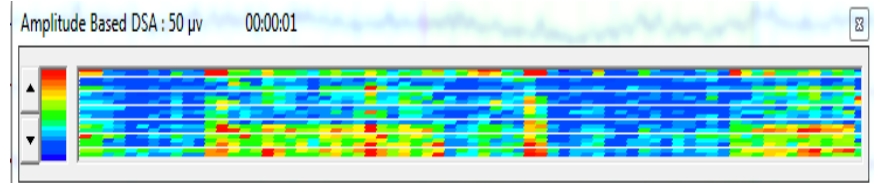

Fig.3. Amplitude based DSA 
Thereafter the MSBE features at each sub band are extracted using entropy analysis. The table I summarizes the MSBE values for three classes of EEG data. The complexity measures decreases for epileptic seizure pattern, so we attain lowest, moderate and higher values of bubble entropy for pre seizure, seizure and normal EEG respectively

Table- I: Bubble entropy parameters for different sub bands

\begin{tabular}{|c|c|c|c|}
\hline \multirow{2}{*}{ Sub-bands } & \multicolumn{3}{|c|}{ Bubble entropy parameter } \\
\hline & Pre seizure event & Seizure event & Normal event \\
\hline DELTA & 10.31 & 11.52 & 12.26 \\
\hline THETA & 12.25 & 13.17 & 17.61 \\
\hline ALPHA & 10.76 & 11.34 & 13.55 \\
\hline BETA & 11.14 & 12.32 & 12.45 \\
\hline GAMMA & 11.27 & 11.31 & 11.38 \\
\hline
\end{tabular}

Classification performance was assessed by measuring standard metrics: Accuracy and Sensitivity.

TP-Seizure event correctly identified as seizure.

TN- Non seizure event correctly identified as non seizure.

FP- Non seizure event incorrectly identified as seizure.

FN- Seizure event incorrectly identified as non seizure.

$$
\text { Accuracy }=(\mathrm{TP}+\mathrm{TN}) /(\mathrm{TP}+\mathrm{TN}+\mathrm{FP}+\mathrm{FN})
$$$$
\text { Sensitivity }=(\mathrm{TP}) /(\mathrm{TP}+\mathrm{FN})
$$

Our result shows that, proposed method provides accuracy of $99.63 \%$, sensitivity of $99.1 \%$. To elaborate the efficiency of our proposed novel technique, a comparison of proposed technique with existing studies is presented in table II, with same dataset used in this paper and hence results are comparable. Now we are able to answer that when we used these integrated feature vectors of MSBE and frequency spectral analysis, then ANN classifier is able to perform classification task with good accuracy.
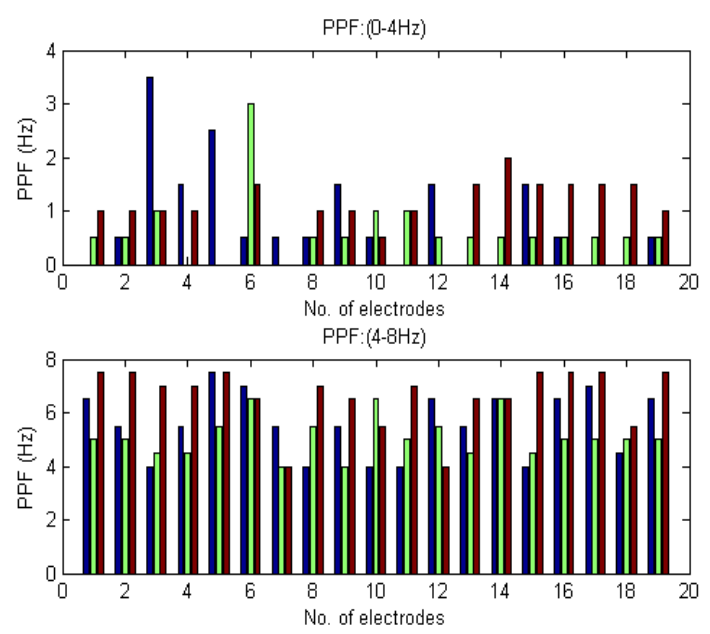

Fig.4. Peak Power frequency of $\delta$ and $\theta$ band
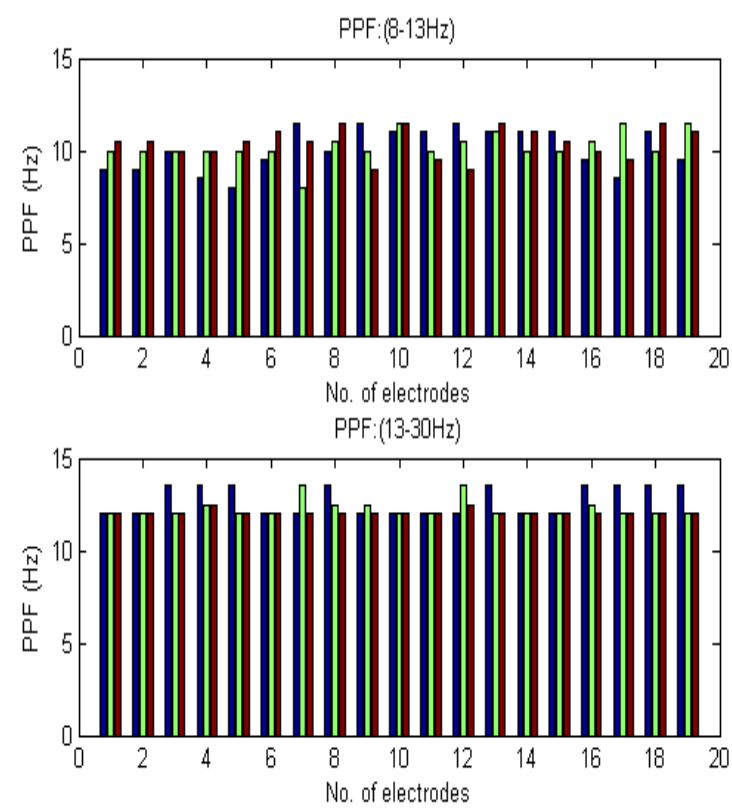

Fig. 5. Peak Power frequency of $\alpha$ and $\beta$ band

Table- II: Performance comparison with the previous works for classification of normal, pre seizure and seizure data

\begin{tabular}{|c|c|c|}
\hline AUTHORS & METHOD & ACCURACY \\
\hline $\begin{array}{c}\text { Ghosh-Dastidar and } \\
\text { Adeli (2007) }\end{array}$ & $\begin{array}{l}\text { Spike neural network } \\
\text { learning algorithms }\end{array}$ & 92.5 \\
\hline $\begin{array}{c}\text { Ghosh-Dastidar et al. } \\
(2007)\end{array}$ & $\begin{array}{l}\text { Wavelet chaos } \\
\text { Methodol ogy with } \\
\text { nonlinear feature }\end{array}$ & 96.7 \\
\hline $\begin{array}{c}\text { Ghosh-Dastidar et al. } \\
\text { (2008) }\end{array}$ & $\begin{array}{c}\text { Wavelet chaos } \\
\text { Methodology with } \\
\text { Principal component } \\
\text { analysis }\end{array}$ & 99.3 \\
\hline $\begin{array}{l}\text { K. C. Chua et al. } \\
\text { (2009) }\end{array}$ & $\begin{array}{l}\text { Higher order spectra } \\
\text { feature with GMM }\end{array}$ & 93.1 \\
\hline Faust et al. (2010) & $\begin{array}{l}\text { Power spectral density } \\
\text { estimation m ethod }\end{array}$ & 93.3 \\
\hline Guo et al. (2011) & $\begin{array}{c}\text { Genetic Programming } \\
\text { using KNN }\end{array}$ & 93.5 \\
\hline $\begin{array}{c}\text { Acharya et al. } \\
\text { (2012a) }\end{array}$ & $\begin{array}{l}\text { Entropy, Higher order } \\
\text { spectra, nonlinear features } \\
\text { Fuzzy sugeno }\end{array}$ & 99.7 \\
\hline Martis et al. (2012) & $\begin{array}{l}\text { Empirical mode } \\
\text { Decomposition with } \\
\text { Hilbert tranform }\end{array}$ & 95.3 \\
\hline Swami et al. (2015) & $\begin{array}{l}\text { 3D phase trajectories with } \\
\text { PNN }\end{array}$ & 97.7 \\
\hline Swami et al. (2016) & Energy and STD & 99.35 \\
\hline Ashwani et al. (2017) & Histogram analysis & 99.3 \\
\hline $\begin{array}{l}\text { Bhattavharyya et al. } \\
\text { (2017) }\end{array}$ & $\begin{array}{l}\text { Multi scale entropy } \\
\text { analysis }\end{array}$ & 99.4 \\
\hline Proposed method & $\begin{array}{l}\text { Multi scale bubble entropy } \\
\text { analysis } \\
\text { Frequency spectral } \\
\text { analysis }\end{array}$ & 99.63 \\
\hline
\end{tabular}




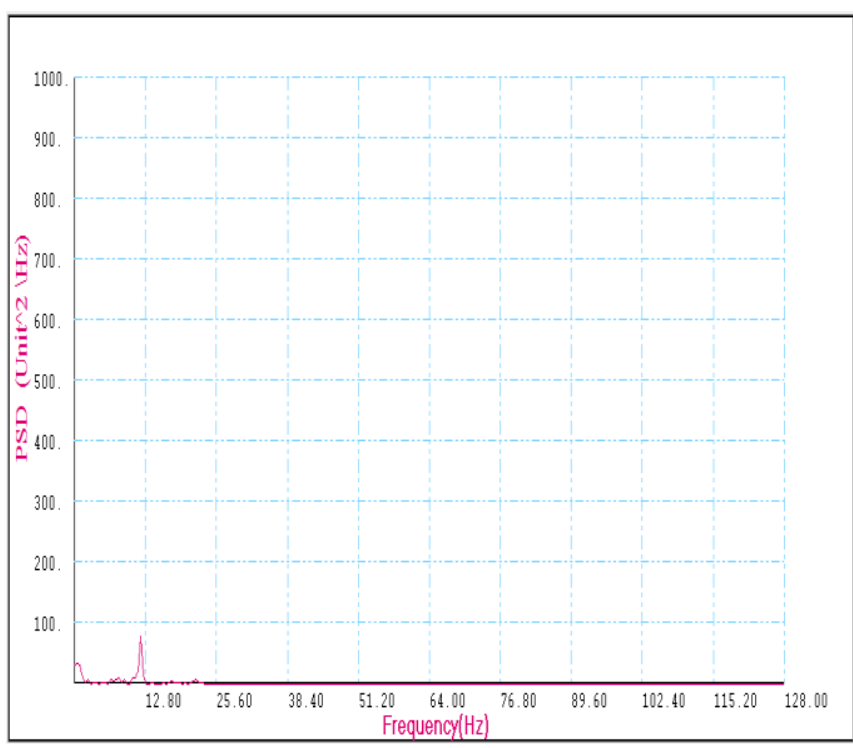

Fig. 6. Power spectral density of normal event

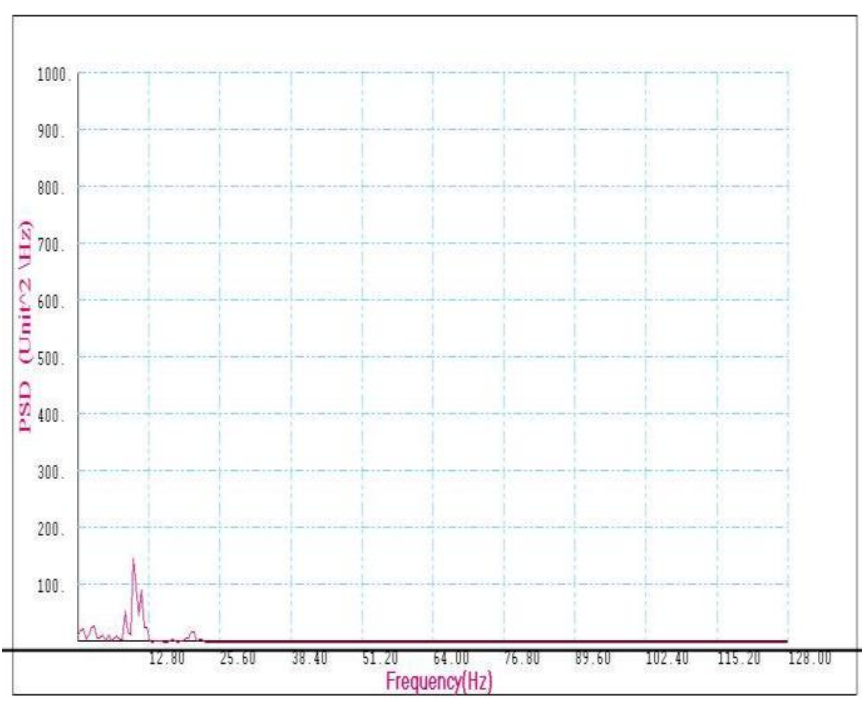

Fig.7. Power spectral density of seizure event

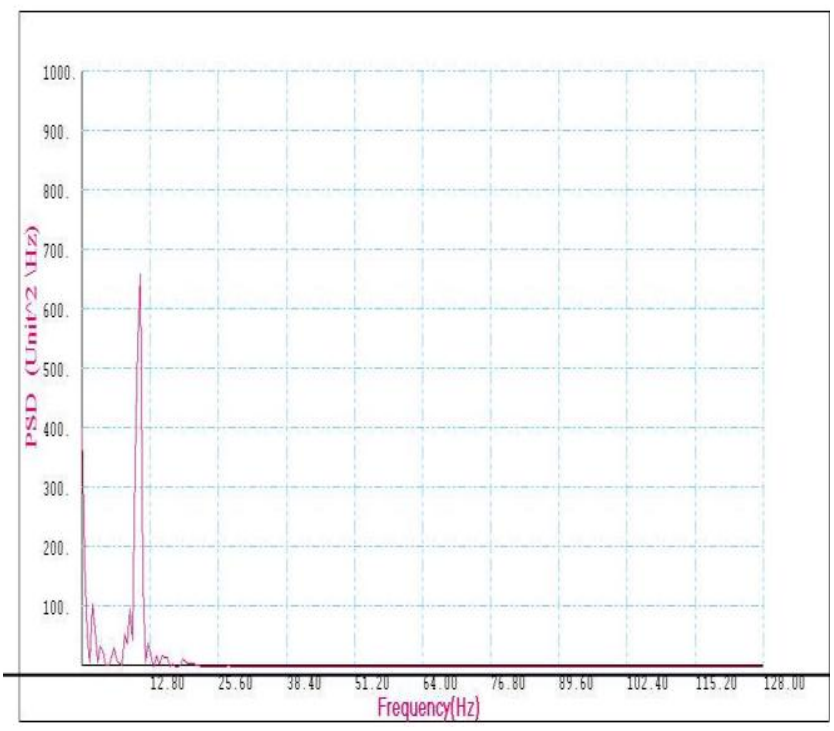

Fig.8. Power spectral density of Pre seizure event

\section{CONCLUSION}

In this paper, an automated method for detection of epileptic seizure has been proposed that offers different level of complexity measures and remarkable stable performance. The proposed technique take into account both frequency spectral analysis and MSBE analysis for construction of integrated feature set. We have demonstrated the capability of multimodality feature analysis for differentiating three classes of EEG (normal, pre seizure and seizure). The performance results shows that our approach provides classification accuracy of $99.63 \%$ and sensitivity of $99.1 \%$

\section{REFERENCES}

1. Y.U. Khan et al., "Wavelet based automatic seizure detection in intracerebral Electroencephalogram", in Clinical neurophysiology, 2003, vol. 114, pp. 898-908.

2. N. Kannathala et al., "Nonlinear analysis of EEG signals at different mental states", in Biomedical online journal, 2004, vol. 3(7), pp. 3-7.

3. N. Kannathala et al.,"Entropies for detection of epilepsy in EEG, Computer Methods and Programs in Biomedicine", 2005, vol. 80, pp. 187-194.

4. Abdulhamit Subasi et al., "Epileptic seizure detection using dynamic wavelet network", in Expert Systems with Applications, 2005, vol. 29, pp. 343-355.

5. V. Srinivasan et al., "Artificial neural network based epileptic detection using timed domain and frequency-domain features", in Journal of medical systems, 2005, vol. 29(6).

6. Ghosh-Dastidar et al., "Improved spiking neural networks for EEG classification and epilepsy and seizure detection", in Integrated computer aided engineering, 2007, vol. 14(3), pp. 187-212.

7. Ghosh-Dastidar et al., "Mixed-band wavelet-chaos-neural network methodology for epilepsy and epileptic seizure detection", in IEEE transactions on biomedical engineering, 2007, vol. 54(9), pp. 1545-1551..

8. Vairavan Srinivasan et al., "Approximate entropy based epileptic EEG detection using artificial neural networks", in IEEE transaction on information technology in biomedicine, 2007, vol. 11(3).

9. Alexandros et al., "Epileptic seizure detection in EEGs using time frequency analysis", in IEEE transaction on information technology in biomedicine, 2009, vol. 13(5).

10. Ghosh-Dastidar et al., "A new supervised learning algorithm for multiple spiking neural networks with application in epilepsy and seizure detection", in Neural networks, 2009b, vol. 22, pp. 1419-1431.

11. K. C. Chua et al., "Automatic identification of epileptic electroencephalography signals using higher-order spectra", in Proceedings of the institution of mechanical engineers, Journal of engineering in medicine, 2009, vol. 223(4), pp. 485-495.

12. K. C. Chua et al., "Analysis of epileptic EEG signals using higher order spectra", in Journal of medical \& engineering technology, 2009a, vol. 33(1), pp. 42-50.

13. Hasan Ocak et al., "Automatic detection of epileptic seizures in EEG using discrete wavelet transform and approximate entropy", in Expert systems with applications, 2009, vol. 36, pp. 2027-2036.

14. Guo et at., "Automatic feature extraction using genetic programming, An application to epileptic EEG classification", in Expert systems with applications, 2011, vol. 38, pp. 10425-10436.

15. Martis. et at., "Application of empirical mode decomposition (EMD) for automated detection of epilepsy using EEG signals", in International journal neural systems, 2012, vol. 22(6), pp. 1-16.

16. Acharya et al., "Application of non-linear and wavelet based features for the automated identification of epileptic EEG signals", in International journal of neural systems, 2012a, vol. 22(2), pp. 1-14.

17. Bhattacharyya et al., "Tunable-Q wavelet transform based multi scale entropy measure for automated classification of epileptic EEG signals", in Application of signal processing methods for systematic analysis of physiological health, 2017b.

18. Bhattacharyya et al., "A multivariate approach for patient-specific EEG seizure detection using empirical wavelet transform", in IEEE transaction on biomedical engineering, 2017a, vol. 64(9), pp. 2003-2015. 
19. George Manis et al., "Bubble entropy: An entropy almost free of parameter", in IEEE transaction on biomedical engineering, 2017, vol. 64(11), pp. 2711-2718.

20. 20 Ashwani Kumar Tiwari et al., (2017), Automated diagnosis of epilepsy using key point based local binary pattern of EEG signals, IEEE transaction on biomedical health Information, 2017, vol. 21(4), pp. 888-896.

21. Faust et al., "Automatic identification of epileptic and background EEG signals using frequency domain parameters", in International journal of neural systems, 2010, vol. 20(2), pp. 159-176.

22. R. Yadav et al., "Morphology based automatic seizure detector for intracerebral EEG recordings", in IEEE transaction on biomedical engineering, 2012, vol. 59(7)

23. Ali Shahidi Zandi et al., "Automated real time epileptic seizure detection in scalp EEG recordings using an algorithm based on wavelet packet transform", in IEEE transactions on biomedical engineering, 2010, vol. 57(7).

24. Subasi et al., "EEG signal classification using wavelet feature extraction and a mixture of expert model", in Expert systems with applications, 2007, vol. 32(4).

25. J. Mitra et al., "A multistage system for the automated detection of epileptic seizures in neonatal electroencephalography”, in Journal of clinical neurophysiology, 2009, vol. 26 (4).

\section{AUTHORS PROFILE}

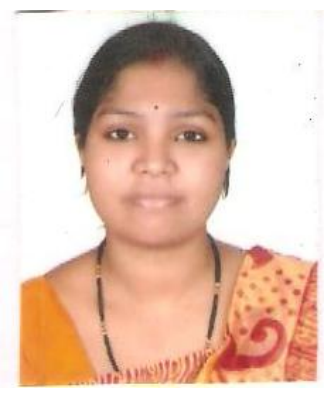

Hemlata Pal received the BE degree in Electronics and communication from Medicaps University, Indore, India, in 2007, the ME degree in Electronics and communication from SGSITS Indore, India in 2011. She is currently pursuing $\mathrm{PhD}$ from School of Electronics, Devi Ahilya University, Indore. She is an Assistant Professor with IET, Devi Ahilya University, Indore. Her current research interests include biomedical signal processing, medical image processing, compression of biomedical signal, Digital signal processing. 